$01 ; 14$

\title{
Оценки вариабельности сердечного ритма методом рекуррентных графиков
}

\author{
() В.Л. Гиляров
}

Физико-технический институт им. А.Ф. Иофре РАН, Санкт-Петербург, Россия

E-mail: Vladimir.Hilarov@mail.ioffe.ru

\section{Поступило в Редакцию 13 марта 2018 г.}

Впервые проведен анализ вариабельности сердечного ритма людей в зависимости от их возраста методом числовых характеристик рекуррентных графиков. Показано, что результаты сопоставимы с данными, которые получаются методом мультифрактального анализа. Отмечено, что метод рекуррентных графиков обладает значительным преимуществом, поскольку требует значительно меньшего числа временны́х отсчетов, и поэтому может быть применен для экспрессдиагностики в экстремальных ситуациях.

DOI: 10.21883/PJTF.2018.23.47013.17286

Частота сердечных сокращений (ЧСС) человека и животных зависит от множества факторов, таких как пол, вес, возраст, сердечные заболевания, стресс, физическая активность и т.д. При этом изменения ЧСС могут происходить прямо в процессе измерения, например при кардиомониторинге. Наличие нестационарности во временны́х рядах различных физиологических характеристик (частота дыхания, RR-интервалы) затрудняет анализ этих временнб́х рядов, поскольку стандартные статистические методы, такие как расчет моментов распределений, корреляционных функций и спектра мощности, для нестационарных рядов неприменимы [1]. Действительно, средние величины (математическое ожидание, дисперсия, ковариация) для нестационарных рядов непостоянны. Это приводит к тому, что теоремы о состоятельности и асимптотической нормальности выборочных оценок и их дисперсий не выполняются. Для различных выборок одинаковой длины функции распределения разные. При увеличении размера выборок точность оценок не возрастает. 
Корреляционные функции нестационарного ряда зависят не от временно́го лага, но от каждого из времен по отдельности, а спектр мощности не может быть вычислен на основе теоремы о свертке и является двухчастотным. Более того, для нелинейных процессов спектральный анализ лишен вообще какого-либо смысла, поскольку принцип суперпозиции для таких процессов не выполняется.

Для изучения нестационарных временны́х рядов было предложено использовать различные мультифрактальные методики [1-6]. Было обнаружено, что ширина спектра сингулярностей $f(\alpha)$ уменьшается с возрастом и при различных сердечных заболеваниях. Эта тенденция трактуется как снижение адаптивной способности индивидуума [1]. С точки зрения нелинейной динамики такое поведение обусловлено изменением характера процесса от более сложного мультифрактального к более простому - монофрактальному, т.е. фрактальной самоорганизацией. Мультифрактальные методы предполагают использование временны́х рядов значительной длины для того, чтобы их можно было анализировать в широком диапазоне масштабных уровней. Значительно меньшая длина ряда требуется для построения рекуррентных графиков и количественного рекуррентного анализа [7]. В настоящей работе метод рекуррентных графиков используется для оценки вариабельности ЧCC.

Расчет проводился для здоровых мужчин с нормальным синусовым ритмом из базы данных Physionet [8]. Из суточных измерений вырезались первые 1800 отсчетов с целью сравнения результатов с данными, полученными методом фотоплетизмографии. На основе исходных данных строились рекуррентные матрицы вида

$$
R_{i, j}\left(\varepsilon_{i}, m\right)=\theta\left(\varepsilon_{i}-\left\|\mathbf{X}_{i}(m)-\mathbf{X}_{j}(m)\right\|\right),
$$

где $\mathbf{X}_{i}(m)$ - фазовая траектория динамической системы в $m$-мерном фазовом пространстве, $\theta$ - функция Хевисайда, а $\|$ || - норма вектора. Рекуррентная матрица, построенная в виде графика, представляет собой двумерное множество точек, отвечающих соотношению (1), состоящее из нулей и единиц, причем близкие (рекуррентные) точки принято отображать черным цветом, а далекие - белым. Близость соседей точки $i$ определяется параметром $\varepsilon_{i}$. Выбор больших величин размерности пространства вложения $m$ необходим в случае восстановления аттрактора исходя из теоремы Такенса для скалярных рядов. Так

Письма в ЖТФ, 2018, том 44, вып. 23 
как в данной работе такая процедура не используется, выбиралось значение $m=1$, поскольку, как показано в обзоре [7], рекуррентные матрицы со значением $m>1$ могут быть выведены из матрицы с $m=1$. Выбор величины порога $\varepsilon_{i}$ существенно влияет на результаты, поэтому обсуждается во многих работах, список которых приведен в [7]. Если выбрать параметр $\varepsilon_{i}$ слишком большим, то точки в основном окажутся близкими друг к другу (рекуррентными), и график будет представлять собой поле черного цвета, а если слишком малым, то рекуррентных точек практически не будет. Согласно рекомендациям [7], величина $\varepsilon_{i}$ выбиралась из условия постоянства плотности рекуррентных точек, равной 0.1 (так называемый $F A N$-критерий). Для расчетов применялся свободно распространяемый пакет программ CRP Toolbox [7].

На рис. 1, $а$ приведена зависимость от возраста параметра рекуррентного графика, называемого детерминизмом $(D E T)$. Эта величина определяется следующим образом. Сначала вводится в рассмотрение уровень возврата

$$
R R\left(\varepsilon_{i}\right)=\frac{1}{N^{2}} \sum_{i, j=1}^{N} R_{i j}\left(\varepsilon_{i}, m\right) .
$$

Он представляет собой плотность рекуррентных точек на графике. Поскольку в настоящей работе используется алгоритм $F A N$, сохраняющий постоянной плотность рекуррентных точек, величина $R R \approx 0.1$. Величина детерминизма вводится как отношение числа рекуррентных точек, формирующих диагональные линии, параллельные главной диагонали, к полному числу рекуррентных точек:

$$
D E T=\frac{\sum_{i, j=1}^{N} D_{i j}}{\sum_{i, j=1} R_{i j}} .
$$

Отметим, что стохастические, слабо коррелированные процессы вообще не имеют диагональных линий, либо эти линии очень короткие, в то время как детерминированные процессы имеют более длинные диагонали и меньшее число изолированных рекуррентных точек [7]. Из рис. 1, $a$ видно, что величина детерминизма в среднем увеличивается с увеличением возраста человека.

На рис. 1, $b$ приведена зависимость от возраста максимальной диагональной линии рекуррентного графика $L_{\max }$. Эта величина связана

Письма в ЖТФ, 2018, том 44, вып. 23 

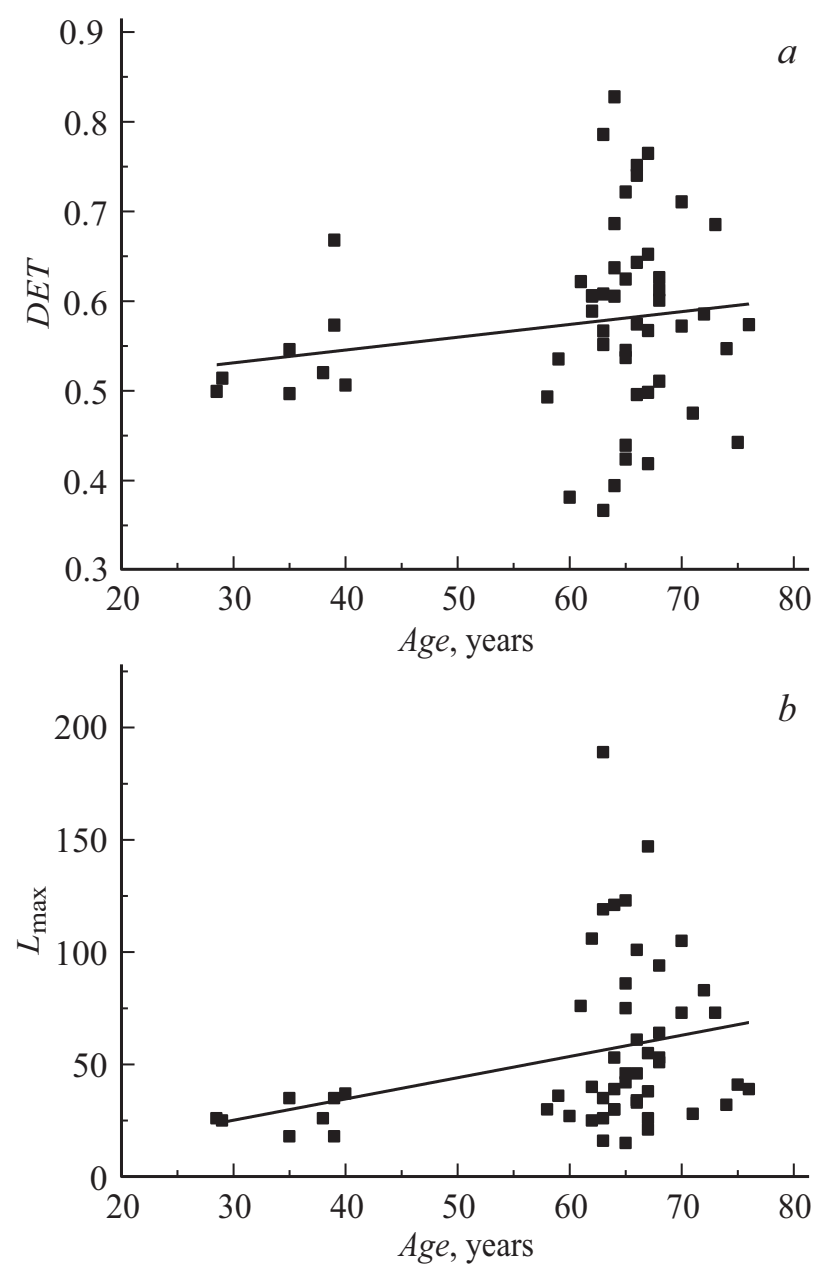

Рис. 1. Зависимость от возраста детерминизма $D E T(a)$ и максимальной длины диагональной линии $L_{\max }(b)$ по данным из портала Physionet. Прямые линии отвечают линейной регрессии (по методу наименьших квадратов).

с расходимостью во времени траекторий фазового пространства. Чем быстрее расходятся фазовые траектории, тем короче диагональные

6 Письма в ЖТФ, 2018, том 44, вып. 23 
линии рекуррентного графика. Видно, что величина $L_{\max }$ также в среднем увеличивается с возрастом. Увеличение степени детерминизма и $L_{\max }$ с возрастом, представленное на рис. 1 , качественно согласуется с уменьшением ширины мультифрактального спектра, обнаруженным в [1]. Это подтверждает тот факт, что динамика процесса с возрастом имеет тенденцию к упрощению - детерминированности и монофрактальности. Заметим, что такая же ситуация наблюдается в процессе разрушения материалов при приближении к моменту разрушения: мультифрактальные спектры сингулярностей различных физических характеристик сужаются, а параметры рекуррентных графиков демонстрируют увеличение детерминированной составляющей по сравнению со стохастической [9].

Для практического применения рассмотренного метода были проведены измерения временны́х рядов пульсации кровотока на запястье методом оптической фотоплетизмографии с помощью бытового фитнестрекера Xiaomi Mi Band 1s. При таком методе измерения светодиод посылает световой сигнал, который, отражаясь, улавливается фотоприемником. Пульсация кровотока вызывает изменение амплитуды отраженного сигнала, временны́е значения которой, пересчитанные на пульс, записываются в память вычислительного устройства. Такой способ измерения ЧСС, конечно, отличается от измерений по электрокардиосигналу, но несомненно, что пульсация кровотока порождена сердечными сокращениями и может быть использована для косвенной оценки сердечной активности и состояния кровеносных сосудов.

Измерения проводились для четырех здоровых людей различного возраста (13-66 лет) и пола (двух мужчин и двух женщин) в состоянии покоя вскоре после пробуждения. Данные записывались на протяжении $30 \mathrm{~min}$, поэтому временны́е ряды содержат 1800 отсчетов пульса. Зависимость детерминизма от возраста представлена на рис. 2. Так же как и для базы данных Physionet, он увеличивается с увеличением возраста человека.

Таким образом, выявлены корреляции параметров рекуррентных графиков и вариации ЧСС с возрастом человека. Отмечено, что при увеличении возраста временна́я последовательность $R R$-интервалов имеет тенденцию к увеличению детерминированности процесса. Эти результаты согласуются с полученными ранее данными об изменении мультифрактальных спектров сингулярности, для которых уменьшение ширины спектра сингулярностей также свидетельствует о тенденции

Письма в ЖТФ, 2018, том 44, вып. 23 


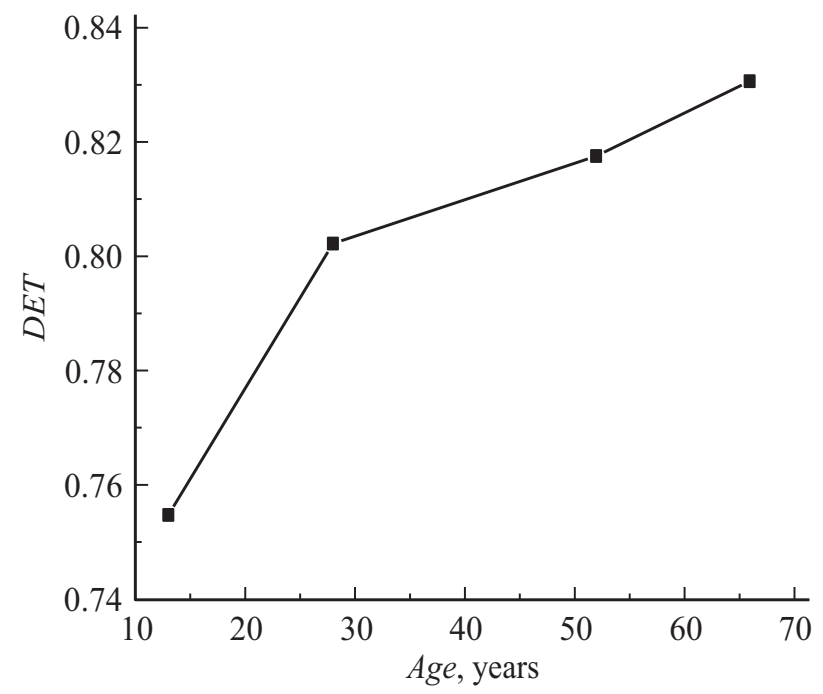

Рис. 2. Зависимость от возраста детерминизма $D E T$ по данным измерений фитнес-трекера.

перехода от сложной мультифрактальной динамики процесса к более простой монофрактальной. Поэтому можно предположить, что метод рекуррентных графиков целесообразно использовать вместо мультифрактального метода для исследования временны́х рядов, полученных на основе различных медицинских измерений. Поскольку для этого метода требуется значительно меньшее число временны́х отсчетов, он может оказаться наиболее эффективным для экспресс-диагностики в экстремальных ситуациях.

Следует отметить, что настоящая работа является постановочной и не претендует на законченное исследование. Статистических данных явно недостаточно для физиологических выводов. Применение многофакторного анализа (метода главных компонент) к параметрам рекуррентных графиков, полученных для базы данных Physionet, показало, что корреляции носят более сложный многомерный характер. Даже первые главные компоненты не определяются однозначно полом и возрастом. Поэтому необходимы дальнейшие систематические исследования вариации ЧСС в конкретных клинических ситуациях.

6* Письма в ЖТФ, 2018, том 44, вып. 23 


\section{Список литературы}

[1] Stanley H.E., Amaral L.A.N., Goldberger A.L., Havlin S., Ivanov P.Ch., Peng C.-K. // Physica A. 1999. V. 270. P. 309-324.

[2] Makowiec D., Galaska R., Dudkowska A., Rynkiewicz A., Zwierz M. // Physica A. 2006. V. 369. P. 632-644.

[3] Sassy R., Signorini M.A., Cerutti S. // Chaos. 2009. V. 19. P. 028507.

[4] Bernaola-Galvan P.A., Romance A.R., Carpena P. // Phys. Rev. E. 2017. V. 96. P. 032218.

[5] Lerma C., Echeverria J.C., Infante O., Perez-Grovas H., Gonzalez-Gomez H. // Chaos. 2017. V. 27. P. 093906.

[6] Castiglioni P., Lazzeroni D., Coruzzi P., Faini A. // Complexity. 2018. V. 2018. P. 4801924 (1-14).

[7] Marwan N., Romano M.C., Thiel M., Kurths J. // Phys. Rep. 2007. V. 438. P. 237-329.

[8] https://www.physionet.org/physiobank/database/nsr2db/

[9] Гиляров В.Л. // ФТТ. 2015. Т. 57. С. 2204-2211. 